\title{
Pediatric pancreatic Burkitt lymphoma with obstructive jaundice: case report of a six-year-old child
}

\author{
Imane Athmani ${ }^{1}$, Marie-Amelyne Le Rouzic ${ }^{1}$, Julie Valduga ${ }^{1}$, Ludovic Mansuy ${ }^{1}$, \\ Audrey Contet ${ }^{1}$, Marie-Agnès Galloy ${ }^{2}$, Fanny Fouyssac ${ }^{1}$, Pascal Chastagner ${ }^{\star}, 1$ \\ 1Department of Pediatric Hematology and Oncology, Children's University Hospital, Nancy, France, \\ ${ }^{2}$ Department of Pediatric Radiology, Children's University Hospital, Nancy, France
}

\begin{abstract}
Introduction: Burkitt lymphoma represents about $50 \%$ of non-Hodgkin lymphomas and $3 \%$ of cancers in children. It commonly affects the abdomen; however, pancreatitis is a very rare initial presentation. Less than 10 cases with this presentation are reported in the literature. Observation: We report the case of a six-year-old girl who presented a rapidly progressing obstructive jaundice evolving for a week. The LDH rate was not increased. Medical imaging has shown a global increase in the size of the pancreas, homogeneous hepatomegaly with dilatation of the gallbladder and the intra-hepatic bile ducts, and infiltration of the left ovary, without ascites. The patient was diagnosed with pancreatic Burkitt Lymphoma after an endoscopic exploration and tumor biopsy. Conclusion: While extremely rare, Burkitt lymphoma should be evoked in the case of pancreatitis with a global increase of the pancreas size, associated with other intra-abdominal organ infiltration in the pediatric population.

Keywords: Burkitt lymphoma, pancreatic tumor, obstructive jaundice, children, chemotherapy
\end{abstract}

\section{Introduction}

Burkitt lymphoma is a high grade B-cell non-Hodgkin lymphoma which often presents with extranodal manifestations and occurs most often in children and immunocompromised hosts. It is one of the fastest growing malignancies in humans. The sporadic form commonly affects the abdomen, and the ileocecal region is the main site of involvement [1-4]. The frequency of pancreatic Burkitt lymphoma among lymphoma of the abdomen is not known because of its rarity. Primary pancreatic lymphoma represents less than $0.5 \%$ of all pancreatic masses in adults. Moreover, acute pancreatitis is a very rare initial presentation of non-Hodgkin lymphomas [5-10]. We present the case of a six-year-old

Received: October 2017; Accepted after review: November 2017; Published: December 2017.

${ }^{*}$ Corresponding author: Prof. Pascal Chastagner Department of Pediatric Hematology and Oncology, Children's University Hospital, 11 Allée du Morvan, 54511 Vandoeuvre, France.

Email: p.chastagner@chru-nancy.fr girl diagnosed with Burkitt lymphoma who presented with obstructive jaundice and pancreatitis.

\section{Case Report}

We report the case of a six-year-old female child who presented with jaundice evolving for one week and who had had abdominal pain and diarrhea for several weeks. She had no fever. The parents described a one-kilogram weight loss over a week, with good overall physical condition. The Lansky score was $100 \%$. The patient had no personal or family medical history, no surgical history, and had never travelled abroad.

The clinical examination found isolated conjunctival icterus and reproducible pain upon palpation of the epigastric region. There was no mass, no liver or spleen enlargement.

Biological characteristics were as follows: hemoglobin: $11.7 \mathrm{~g} / \mathrm{dL}$, total leucocyte count: $5520 / \mathrm{mm}^{3}$, platelet count: $267000 / \mathrm{mm}^{3}$, hepatic cytolysis with AST: $212 \mathrm{IU} / \mathrm{L}$ and ALT: 
$195 \mathrm{IU} / \mathrm{L}$, lipase > $600 \mathrm{IU} / \mathrm{L}$, total bilirubin: $162.1 \mu \mathrm{mol} / \mathrm{L}$, direct bilirubin: $141.9 \mu \mathrm{mol} / \mathrm{L}$, LDH: $285 \mathrm{IU} / \mathrm{L}$, prothrombin time ratio: 0.91, TQ: $86 \%$, fibrinogen: $4.7 \mathrm{~g} / \mathrm{L}$, blood sugar level: $84 \mathrm{mg} / \mathrm{dL}$, uric acid: $27 \mathrm{mg} / \mathrm{L}$, creatinine: 9 $\mathrm{mg} / \mathrm{L}$, potassium: $5 \mathrm{mmol} / \mathrm{L}$. Serological tests for EBV, CMV, HAV, HBV, HCV, VIH 1 and 2, HSV 1 and 2 were negative, as were PCR results.

Abdominal ultrasound showed a dilatation of both the extra- and intra-hepatic bile ducts. The pancreas head was shifted forward and laterally by a nodular mass measuring 34 x 33 $\mathrm{mm}$. It also showed a homogenous hepatomegaly with regular outlines $(145 \mathrm{~mm})$, a normal-sized and homogeneous spleen and a retro-vesical elongated solid mass $(70 \times 30$ $\mathrm{mm}$ ) being attached to the uterus, with a thin layer of intraperitoneal effusion around this pelvic mass.

An abdominal and pelvic CT scan was performed which revealed a global enlargement of the pancreas with a pseudotumoral aspect. It confirmed a homogeneous hepatomegaly with dilatation of the gallbladder and the intra-hepatic bile ducts. The extrahepatic bile duct was dilated and ended brutally in the enlarged pancreas head. The retro-uterine pelvic mass was located in front of the rectum. No bone lesion was detected.

An abdominal MRI showed a global increase in the size of the pancreas without pancreatic duct dilatation. At the back of the womb and in front of the rectum, the heterogeneous mass was measuring $75 \times 53 \times$ $37 \mathrm{~mm}$ (Figure 1).
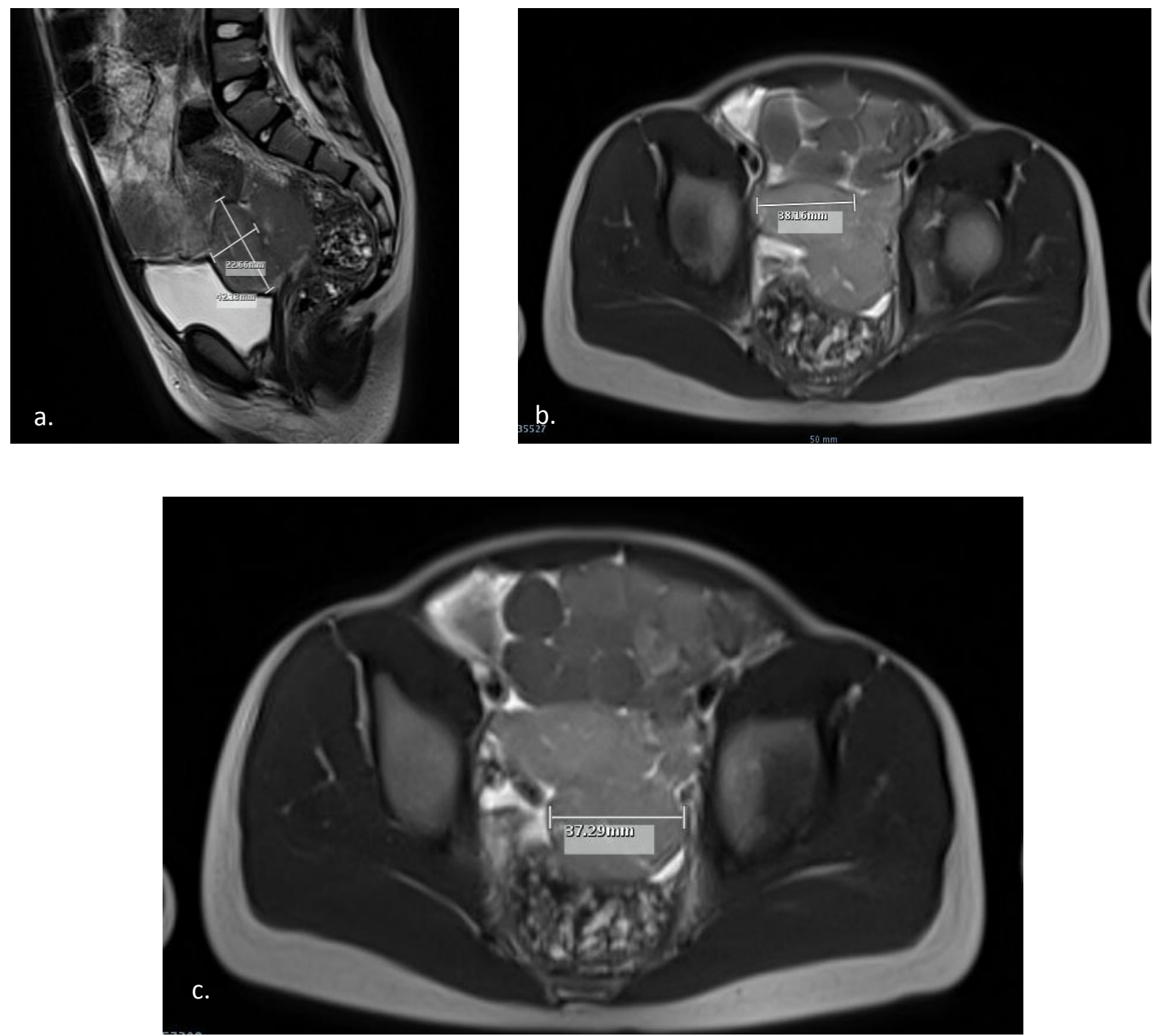

Fig. 1. Sagittal (a) and axial (b, c) sections of pelvic MRI showing a retro-uterine pelvic mass located in front of the rectum and measuring $75 \times 53 \times 37 \mathrm{~mm}$. 
Endoscopic exploration revealed that the retro uterine mass was an increased-size ovary most likely due to a tumoral infiltration. There was no ascites. Tumor biopsy samples were performed during gastrointestinal endoscopy.

Histopathological examination confirmed the diagnosis of Burkitt lymphoma. The lymphoid cells expressed CD45, CD20, CD79a, C MYC, CD10, BCL6 but were negative for BCL2, CD34, CD30, MUM1. Tumor proliferative activity (Ki-67 expression) was estimated at around $100 \%$ in lymphoid cells. There was no expression of WT1, desmin, TdT, myogenin or CD99. Cytogenetic testing revealed a c-MYC gene rearrangement in 15 to $20 \%$ of tumor cells.

Moreover, a thoracic and cervical CT scan showed no lymphadenopathy. A bone marrow aspiration showed a normocellular marrow, with rare undifferentiated cells but no blast cells. A bone marrow biopsy and a cerebrospinal fluid assessment were performed and yielded normal results. The PET scan (performed after 9 days of steroids therapy) showed no suspicious uptake of ${ }^{18} \mathrm{~F}$ FDG.

As a result of the above investigation, the patient was diagnosed with pancreatic Burkitt lymphoma associated with an infiltration of the left ovary. She was classified as stage IV of the Saint Jude system and treated in group $B$ according to the recommendations of Inter BNHL-Ritux 2010 protocol.

At day 7 of the induction treatment (vincristine, cyclophosphamide, steroids and intrathecal methotrexate + hydrocortisone), the patient presented a regression of conjunctival icterus and a decrease in abdominal pain. Blood tests showed nearly complete normalization of hepatic function and lipase level.

Abdominal ultrasound showed a decrease in the pancreas size (head: $4 \times 21 \times 31 \mathrm{~mm}$; body: $14 \mathrm{~mm}$; tail: $23 \mathrm{~mm}$ ) and a decrease in the infiltration of the left ovary $(41 \times 27 \times 18$ $\mathrm{mm})$. After having completed the entire chemotherapy regimen, she is in complete remission and in very good health.

\section{Discussion}

Non-Hodgkin lymphoma accounts for 40 to $50 \%$ of all lymphomas in children and adolescents. It represents approximately 6\% of all malignancies in patients aged 0 to 19 years though Burkitt lymphoma comprises the large majority of primary pediatric gastrointestinal tract lymphomas. It is a highly aggressive non-Hodgkin B-cell lymphoma that often presents at extranodal sites.

In the pediatric population, the more frequent presentation of Burkitt lymphoma is that of a large painful and rapidly growing mass of the abdomen with ascites. Initial workup and staging assess the possibility of kidney, testis/ovary, bone marrow and central nervous system involvement. General evaluation must be done very rapidly because of the very short tumor-doubling time of these lymphoma cells (about 25 hours), in particular evaluating the risk of tumor lysis syndrome which could lead to acute renal failure.

Burkitt lymphoma with isolated involvement of pancreas is very rare since less than 10 cases were reported in the literature [11]. It can present as diffuse enlargement of the entire pancreas gland, caused by tumorinduced pancreatitis [12]. Obstructive jaundice is also infrequent [13].

Burkitt lymphomas are highly responsive to specifically designed multi-agent chemotherapy regimens which begin with low dose of vincristine, cyclophosphamide and steroids, aiming at avoid the tumor lysis syndrome. They are followed by intensive courses including rotations of vincristine, doxorubicine, cyclophosphamide, etoposide and high dose methotrexate and cytarabine for the central nervous system prophylaxis, associated with intrathecal injections of methotrexate. It has been demonstrated that the anti-CD20 monoclonal antibody (Rituximab), when added to these regimens, still improved the prognosis.

The reported case is particular because, while clinical signs were jaundice and abdominal pain, there was no palpable abdominal mass and no ascites which are 
frequent signs at diagnosis of Burkitt lymphomas. Moreover, LDH and uric acid levels were in normal range. Finally, there was an ovary infiltration which initially led to evoke a primary tumor of the ovary, such as malignant germinoma, with metastases within the pancreas.

The diagnosis confirmation was made after histological analysis of pancreas biopsy. The pelvic mass was characterized as being an infiltration of the left ovary. No biopsy was performed on it due to diagnostic uncertainties as well as to avoid spreading tumor cells in the peritoneal cavity in case it wasn't a lymphoma. Before the histopathological diagnosis was made, the patient received steroids therapy in order to decrease the tumor mass and, thus, to reduce the extrinsic compression on the bile ducts. The biopsy and the bone marrow aspiration were performed after 4 days of steroids therapy: some undifferentiated cells were seen in the bone marrow. As a

\section{References}

1. Grajo JR, Mark L. Kayton ML, Steffensen TS, et al. Presentation of ileal Burkitt lymphoma in Children. J Radiol Case Rep 2012; 6:27-38.

2. Kassira N, Pedroso FE, Cheung MC, et al. Primary gastrointestinal tract lymphoma in the pediatric patient: review of 265 patients from the SEER registry. $J$ Pediatr Surg 2011; 46:1956-1964.

3. Francis IR, Glazer GM. Burkitt's lymphoma of the pancreas presenting as acute pancreatitis. J Comput Assist Tomogr 1982; 6:395-397.

4. Merkle EM, Bender G, Brambs HJ. Imaging finding in pancreatic lymphoma: differential aspects. Am J Roentgenol 2000; 174:671-675.

5. Koca T, Aslan N, Dereci S, et al. Burkitt lymphoma with unusual presentation: Acute pancreatitis. Pediatr Int 2015; 57:775-777.

6. Wang YJ, Jeng CM, Wang YC, et al. Primary pancreatic Burkitt's lymphoma mimicking carcinoma with obstructive jaundice and very high CA19-9. Eur J Gastroenterol Hepatol 2006; 18:537-540.

7. Mahajan V, Qian YW, Blake B, et al. Sporadic Burkitt lymphoma presenting as acute consequence, the patient was classified as stage IV of the St Jude system.

\section{Conclusion}

Burkitt lymphoma should always be considered in differential diagnosis of the pancreatitis in children presenting with a solid pancreatic mass. No imaging is able to distinguish pancreatic lymphoma from other types of masses. A rapid biopsy is an accurate measure of diagnosis aiming at starting chemotherapy promptly. The prognosis is very favorable.

\section{Conflict of interest/Funding Statement}

The authors have no conflict of interest to declare.

pancreatitis, concurrent sinusitis, and enlarged adenoids. Case Reports in Pediatrics 2016; 2016:3862175. doi: 10.1155/2016/3862175.

8. Akil M, Kaya A, Selcuk Bektas M, et al. Burkitt's lymphoma causing acute pancreatitis in a child. $J$ Acute Dis 2013; 2:159-160.

9. Aftandilian CC, Friedmann AM. Burkitt lymphoma with pancreatic involvement. $J$ Pediatr Hematol Oncol 2010; 32:338-340.

10. Amodio J, Brodsky JE. Pediatric Burkitt lymphoma presenting as acute pancreatitis: MRI characteristics. Pediatr Radiol 2010; 40:770-772.

11. Nistala SS, Sawalakhe NR, Thiruvengadam $\mathrm{NR}$, et al. A rare case of primary Burkitt lymphoma in a young indian male. Case report and Review of the Literature. J Pancreas 2009; 10: 686-689.

12. Novoselac VA, Katz RJ, Lessen DS, et al. Burkitt's lymphoma arising in the pancreas. J Clin Oncol 2005 ; 23(16 Suppl):6740.

13. Saglam M, Yilmaz $M$, Refik $M M$, et al. A case of pancreatic Burkitt lymphoma: radiological findings. Diagn Interv Radiol 2009, 15:39-42. 